\title{
Organic Field-Effect Transistors Using Hetero-Layered Structure with OLED Materials
}

\author{
Ken-ichi Nakayama, Yong-Jin Pu, Junji Kido and Masaaki Yokoyama \\ Yamagata University, Osaka University
}

Japan

\section{Introduction}

In recent years, organic transistors have attracted much attention due to their advantages in developing low-cost, flexible, and large-area production. So far, many kinds of organic materials have been reported to achieve high-performance organic field-effect transistors (OFETs). There are two types of organic semiconductors, p-type and n-type, whose majority carriers are holes and electrons, respectively. For logic gates application, both types and similar performance OFETs are required for CMOS application. Pentacene is the most popular material in p-type OFET, and many kinds of polymer materials are also reported (McCulloch et al., 2006). On the other hand, the performance of n-type OFETs is generally inferior to that of p-type (Dimitrakopoulos and Malenfant, 2002). In particular, stability in air is the most serious problem in n-type OFET. Fullerene is the most standard n-type material showing the highest mobility (Singh et al., 2007); however, the device cannot operate in air.

There are two guidelines to achieve high mobility and high stability in n-type OFET. One is to develop a new material having deeper LUMO level. Oxygen and water deteriorate OFET performance by accepting electrons from the semiconductor molecule. Therefore, enough deep LUMO level is an efficient way to avoid effect of oxygen or water. In fact, there have been many materials having deep LUMO levels, for example, perylene bisimide compound, fullerene derivatives, fluorinated compounds, and so on.

The other important point is surface treatment of the insulator. The field-effect mobility of the organic semiconductor is strongly affected by the device fabrication process. Various methods on surface treatments have been reported to improve the carrier mobility. The HMDS treatment is a standard and efficient way to make the surface hydrophobic (Lin et al., 1997; Lim et al., 2005). Organic semiconductor can aggregate with high crystallinity on the hydrophobic surface without influence of the substrate surface. These methods were developed in p-type OFETs; however, they are also efficient to improve the mobility and stability of n-type OFETs. Recently, it has been pointed out that low mobility and instability in air of n-type organic semiconductor is attributed to the surface electron traps of the gate insulator, and if electron traps can be perfectly eliminated, almost organic semiconductors can be operate in n-type mode (Chua et al., 2005). Therefore, it has been believed that the gate insulator surface should be as possible as inert to achieve high mobility and stability in n-type OFETs. 
In this chapter, we introduce a new concept of a hetero-layered OFET to improve the performance of OFETs instead of conventional surface treatment methods. The heterolayered OFET includes an interfacial layer of electronic active organic semiconductor having opposite transport polarity between the insulator and channel layer. For the interfacial layer of n-type OFET, we employed various types of hole transporting material, which are generally used for organic light-emitting diodes (OLEDs). For p-type OFET, electron transporting material was employed.

Such a hetero-layered OFETs composed of p-type and n-type organic semiconductors have been studied for ambipolar organic transistors, which aimed at the simple inverter circuit or organic light-emitting transistors (Rost, 2004; Rost et al., 2004). On the other hand, our proposed hetero-layered OFET employs charge transport material of OLEDs. They generally form amorphous films resulting in no FET operation by themselves.

The proposed hetero-layered OFET showed improvement of the mobility compared to the conventional surface treatment. In addition, we found that the stability in air was drastically improved in n-type OFET by using a hole transporting material having higher HOMO level. We discuss the relationship between the OFET performance and the electronic property of the interfacial layer.

\section{Perylene bisimide and hole transporting materials}

In this section, we will introduce the results of perylene bisimide (PTCDI-C8H) for the channel layer and the hole transporting material of NPD, TAPC and m-MTDATA for the interfacial layer. Perylene bisimide compounds are promising n-type organic semiconductor having deep LUMO levels and high crystallinity. In particular, PTCDI with long alkyl chains bring about a highly ordered film structure, and very high electron mobility has been reported (Tatemichi et al., 2006). On the other hand, NPD and TAPC having triphenyl amine structure are very standard hole transporting material for OLED devices. They show comparably high hole mobility and good film formation.

Figure 1 shows the hetero-layered structure OFET with top contact and the molecular structures of m-MTDATA and NPD. Organic transistors were fabricated on a heavily doped Si substrate with $\mathrm{SiO}_{2}$ layer $(300 \mathrm{~nm})$ that works as a common gate electrode. The interfacial semiconductor layer of m-MTDATA and NPD $(20 \mathrm{~nm} \sim 30 \mathrm{~nm})$ were deposited by thermal evaporation. For the comparison, the substrates with well-known surface treatment by octadecyltrichlorosilane (OTS) and hexamethyldisilazane (HMDS) were also prepared. Au source and drain electrodes were deposited through a shadow mask. Channel length and width were defined to be $50 \mu \mathrm{m}$ and $5.5 \mathrm{~mm}$, respectively. The current modulation of OFETs were measured by a semiconductor parameter analyzer in the glove box, where the concentration of oxygen and water were less than $1 \mathrm{ppm}$. The field-effect mobility, threshold voltage and on/off ratio were estimated from the equation of saturation regime, $I_{D}=[(W C \mu) / 2 L]\left(V_{G}-V_{T}\right)^{2}$, where $C$ is the capacitance per unit area of the gate dielectrics, $W$ is the channel width, $L$ is the channel length, $\mu$ is the carrier mobility, and $\mathrm{V}_{\mathrm{T}}$ is the threshold voltage.

Figure 2 shows the transfer characteristics of OFETs with an interfacial layer of NPD, those subjected to HMDS surface treatment, and those without any interfacial layer and not subjected to surface treatment (None). In all the devices, the source-drain current $\left(\mathrm{I}_{\mathrm{D}}\right)$ increased with the positive gate voltage $\left(\mathrm{V}_{\mathrm{G}}\right)$, which indicates that these OFETs operate only in the n-type mode, and the hole-transporting layer does not acts as a p-type channel layer. 
The performances of OFETs with different interfacial layers are summarized in Table 1. The optimum thickness of the interfacial layer is also indicated. The mobility was improved with increasing thickness of the hole transporting layer and showed a maximum around $20 \mathrm{~nm}$. The mobility for heterolayered device was estimated assuming the gate capacitance of only $\mathrm{SiO}_{2}$ because it is difficult to determine the channel interface. The conventional HMDS treatment resulted in an improvement in the mobility from $2.5 \times 10^{-2} \mathrm{~cm}^{2} / \mathrm{Vs}$ (None) to $6.9 \times$ $10^{-2} \mathrm{~cm}^{2} /$ Vs. Interfacial layers composed of NPD and m-MTDATA increased the mobility up to 0.11 and $0.13 \mathrm{~cm}^{2} / \mathrm{Vs}$, respectively.
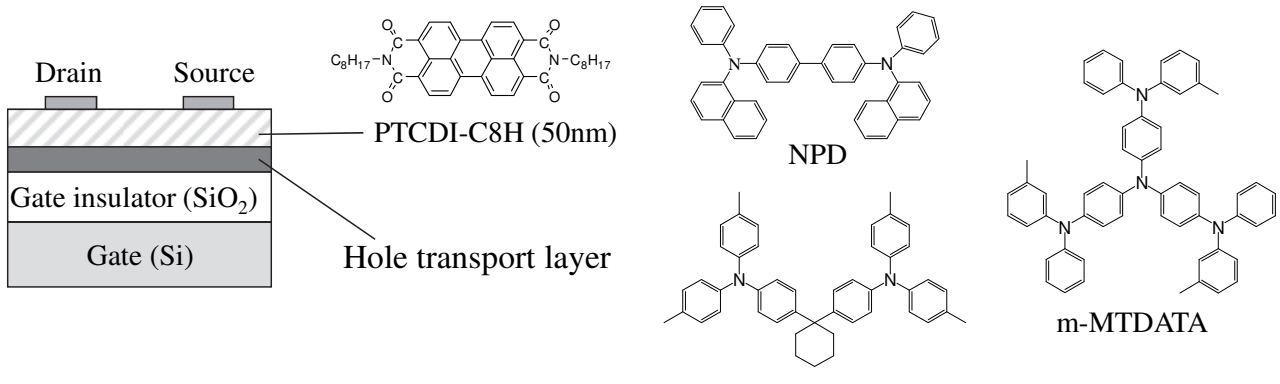

TAPC

Fig. 1. Device structure of hetero-layered OFET using PTCDI-C8H and hole transporting materials.
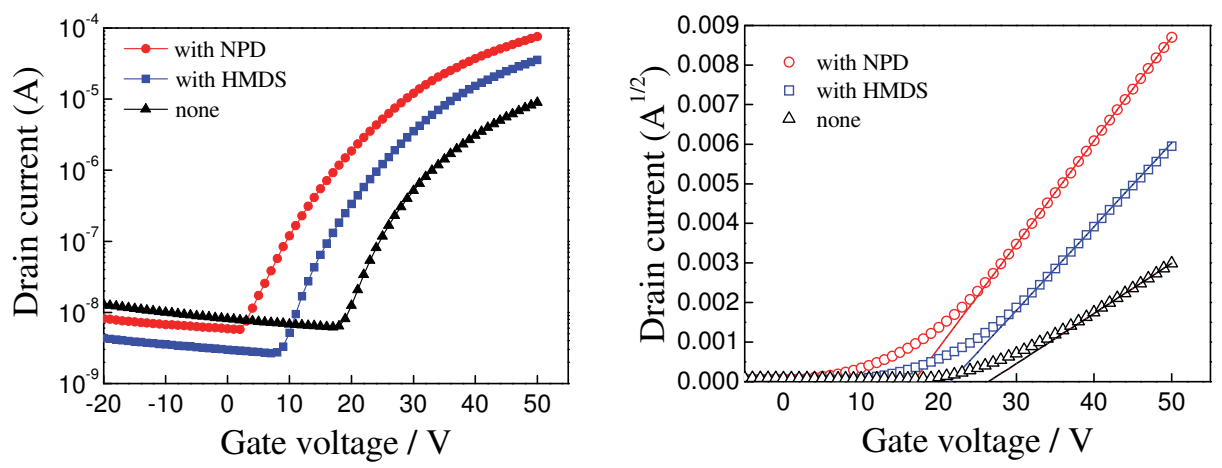

Fig. 2. Transfer characteristics of the n-type OFET with hetero-layered structure and conventional surface treatment.

These results indicate that an electronically active material can be used to fabricate an interfacial layer, and high performance can be achieved without a surface treatment of selfassembly monolayer. We also investigated some other organic materials, n-hexatriacontane that is perfectly inert material, and $\mathrm{Alq}_{3}$ that is a well-known emissive and electron transporting material. In these cases, the mobility was rather reduced to be $10^{-3} \mathrm{~cm}^{2} / \mathrm{Vs}$. These results enable us to conclude that hole transporting materials are responsible for enhancing mobility.

Mobility can also be improved by modifying the structure of the semiconductor film. X-ray diffraction patterns of PTCDI-C8H films with and without the NPD $(10 \mathrm{~nm})$ interfacial layer 
were measured under the same condition (Fig. 3). Patterns of both the films showed a very strong peak at $4.3^{\circ}$ corresponding to $\mathrm{d}=2.05 \mathrm{~nm}$. This peak is assigned to the long axis of the molecules, which are aligned vertically on the surface. However, in the case of the PTCDI-C8H films with the NPD interfacial layer, the diffraction peaks are rather weak, which is also supported by the fact that the high order peaks become unclear, as shown in the magnified inset of Fig. 3. This result indicates that the improvement in mobility caused by the holetransporting interfacial layer is not attributed to the increase in crystallinity of the PTCDI-C8H film. This interpretation is also supported by contact angle measurements. The contact angle of the interfacial layer was $85.8^{\circ}$ for m-MTDATA and $92.5^{\circ}$ for NPD. These values are comparable to that of HMDS-treated $\mathrm{SiO}_{2}$ surface. This fact also indicates that the mobility improvement can be attributed to the electronic effect of hole transporting layer.

\begin{tabular}{cccc}
\hline \hline Surface & $\begin{array}{c}\text { Mobility } \\
\left(\mathrm{cm}^{2} \mathrm{~V}^{-1} \mathrm{~s}^{-1}\right)\end{array}$ & $\begin{array}{c}\text { Threshold } \\
(\mathrm{V})\end{array}$ & $\begin{array}{c}\text { On/off } \\
\text { ratio }\end{array}$ \\
\hline Bare & 0.0351 & 20.4 & $5.18 \times 10^{3}$ \\
HMDS & 0.0690 & 21.5 & $1.32 \times 10^{4}$ \\
n-hexatriacontane $(15 \mathrm{~nm})$ & 0.0407 & 11.8 & $3.00 \times 10^{3}$ \\
TAPC $(20 \mathrm{~nm})$ & 0.0713 & 15.5 & $1.28 \times 10^{4}$ \\
NPD $(10 \mathrm{~nm})$ & 0.110 & 16.7 & $1.17 \times 10^{4}$ \\
m-MTDATA $(20 \mathrm{~nm})$ & 0.127 & 25.3 & $1.33 \times 10^{4}$ \\
Alq $(15 \mathrm{~nm})$ & 0.00686 & 12.4 & - \\
\hline \hline
\end{tabular}

Table 1. Performances of PTCDI-C8H OFETs with different interfacial layers between the gate insulator and the channel layer.

(a) NPD / PTCDI-C8H

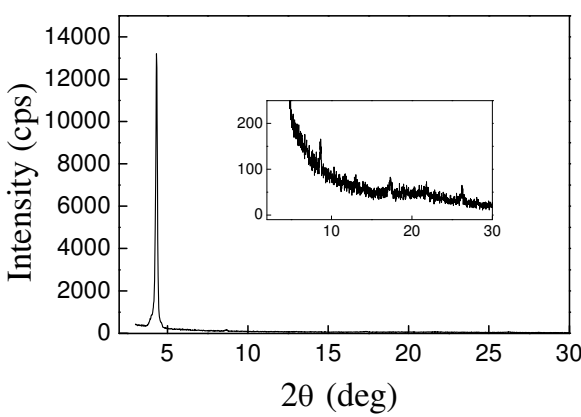

(b) PTCDI-C8H

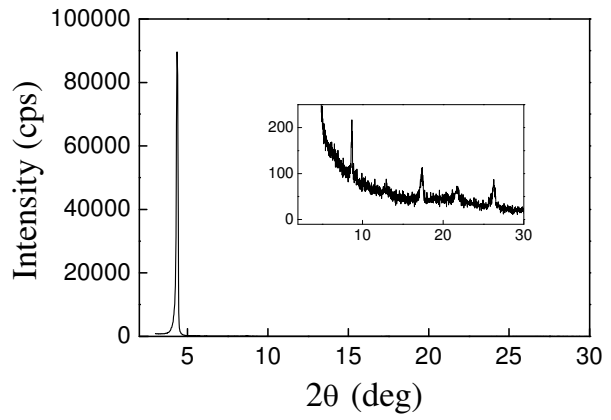

Fig. 3. X-ray diffraction patterns of (a) NPD (10 nm)/PTCDI-C8H (50 nm) film and (b) PTCDI-C8H (50 nm) film deposited on the $\mathrm{Si} / \mathrm{SiO}_{2}$ substrate.

Energy levels (highest occupied molecular orbital (HOMO) and LUMO levels) of the organic semiconductors used in this study are shown in Fig. 4. The n-type organic semiconductor, 
PTCDI-C8H, has a deep LUMO level of $4.6 \mathrm{eV}$. On the other hand, hole transport material of NPD and TAPC has higher HOMO level and wide energy gap exceeding $3 \mathrm{eV}$. Therefore, LUMO level of the interfacial layer is much higher than that of the channel layer.

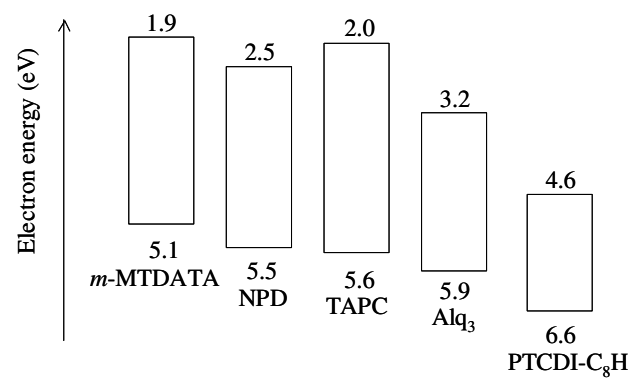

Fig. 4. Energy level diagrams of organic semiconductors used in this study. Upper and lower values indicate the LUMO and HOMO.

These results can be interpreted as following model. Figure 5 shows the schematic energy diagram of the heterolayered OFET. Since the LUMO level of the interfacial layer was considerably higher than that of the n-type semiconductor layer, electrons would not enter the interfacial layer. In addition, the hole-transporting layer did not show p-type FET operation. Therefore, it was concluded that an n-type channel was formed at the interface between the hole-transporting layer and the n-type semiconductor film. The role of the interfacial layer can be basically attributed to the separation of the channel carriers from the surface electron traps, similar to the conventional hydrophobic surface treatment. However, it was noted that the mobility or threshold voltage had a correlation with the HOMO level of the inserted layer. Mobility increased in the order of m-MTDATA > NPD > TAPC, which corresponded to the order of the HOMO levels, i.e., the interfacial layer with a higher HOMO level exhibited better performance. This result suggests that the nature of semiconductor of the interfacial layer affected the electron transportation process at the interfacial channel.

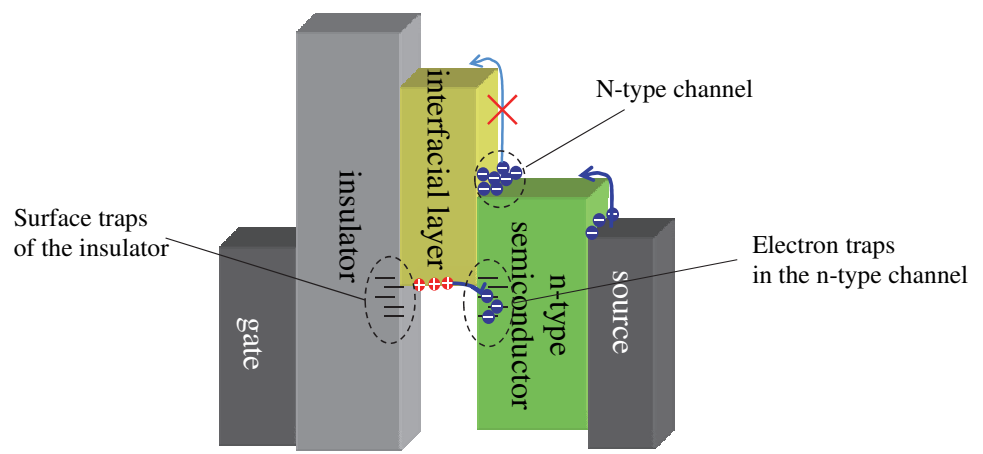

Fig. 5. Schematic relationship of energy levels in the hetero-layered OFET composed of hole transporting layer and n-type organic semiconductors.

This additional effect should be discussed from the viewpoint of electronic interaction between the hole transporting layer and the n-type channel layer. In the single layer device 
of PTCDI-C8H, the surface electron traps of $\mathrm{SiO}_{2}$ can be passivated by inert surface treatment like HMDS. However, there would be many electron traps in the PTCDI-C8H film itself. They cannot be eliminated by surface treatment of the substrates. On the other hand, the hole transporting materials generally have higher HOMO levels, in other words, electron donating character. Therefore, the interfacial layer tends to give electrons toward PTCDI-C8H film at the interface. It may not eliminate the shallow electron traps because the HOMO level of NPD is far from the LUMO level of PTCDI-C8H, but the deep electron traps are expected to be filled in advance by thermally activated charge transfer. As a result, the injected electrons can move smoothly at the interface, resulting in the observed high electron mobility. We conclude that this trap-filling effect is essential of the hetero-layered OFET.

Thus, we proposed the concept of hetero-layered OFETs and ascertained its validity. The performance was improved by insertion of the electronic active material rather than an inert surface treatment. Because the film structure of the deposited PTCDI-C8H was not changed by the surface treatment or interfacial layer, we concluded that this improvement is attributed to electron donating character of the hole transporting layer.

\section{3. $\mathrm{C}_{60}$ and hole transporting materials}

In this section, the concept of hetero-layered OFET is applied to the combination of $\mathrm{C}_{60}$ channel layer and hole transporting material (Fig. 6). $\mathrm{C}_{60}$ is the most standard material of ntype organic semiconductors and the highest performance in n-type OFET has been reported. The device structure is the same structure with the previous section. For the interfacial layer, typical hole transporting material of NPD and m-MTDATA were used.
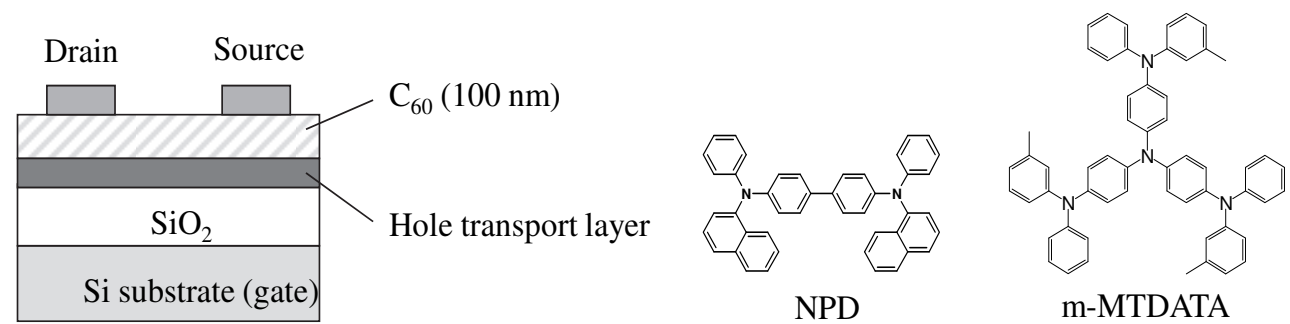

Fig. 6. Device structure of hetero-layered OFET composed of $\mathrm{C}_{60}$ and hole transporting materials.

Figure 7 shows the drain current-gate voltage $\left(\mathrm{I}_{\mathrm{D}}-\mathrm{V}_{\mathrm{G}}\right)$ characteristics of the hetero-layered OFETs with m-MTDATA and NPD interfacial layer, and the single layer $\mathrm{C}_{60}$ OFETs on OTStreated, HMDS-treated, and non-treated substrates. Also in this case, the $I_{D}-V_{G}$ curves for the hetero-layered devices increased only for positively biased gate voltage with almost no hysteresis for forward and backward sweeps. This means that they did not operate as an ambipolar transistor, and the interfacial layer of the hetero-layered device did not work as a p-type channel layer.

The performance of each device were summarized in Table 2. The conventional surface treatment by OTS and HMDS brought about high electron mobility of 0.50 and $0.80 \mathrm{~cm}^{2} / \mathrm{Vs}$, respectively, whereas the normal device on the non-treated substrate showed low mobility of $7.5 \times 10^{-3} \mathrm{~cm}^{2} / \mathrm{Vs}$. However, it should be noted that the hetero-layered device with $\mathrm{m}-$ 
MTDATA and NPD achieved very high electron mobility of 1.1 and $1.8 \mathrm{~cm}^{2} / \mathrm{Vs}$, respectively. These values are the highest value for $\mathrm{C}_{60}$ FETs without any surface treatment or substrate heating. Thus, it was revealed that the hetero-layered OFET is generally efficient to improve the performance even in high performance OFETs using $\mathrm{C}_{60}$.

(a)

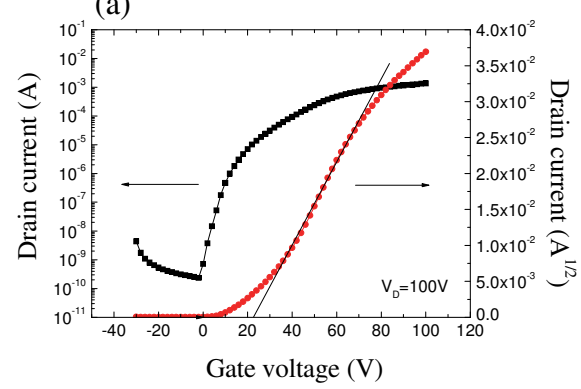

(c)

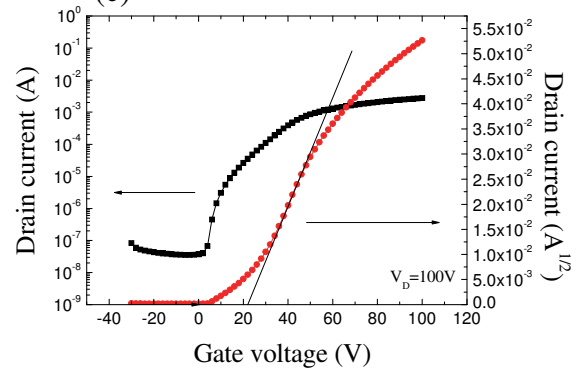

(b)

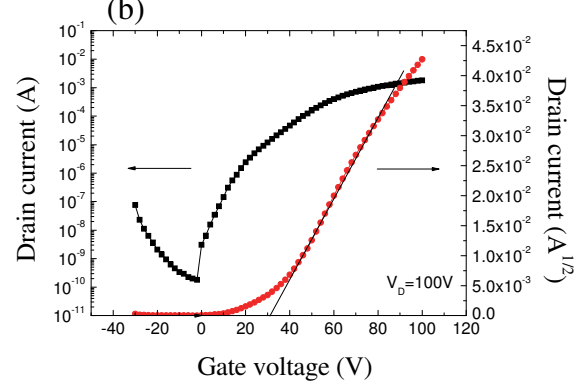

(d)

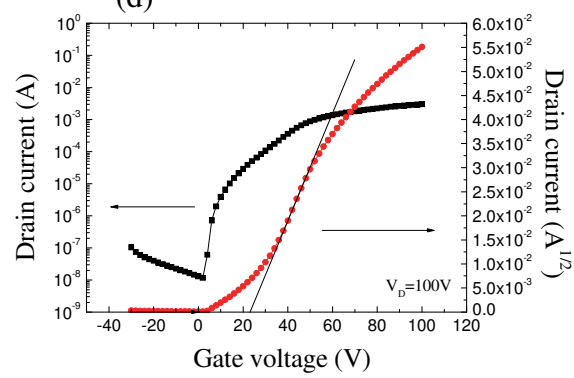

Fig. 7. Transfer characteristics of OFETs devices of $\mathrm{C}_{60}$ film on the various kinds of surface, (a) OTS, (b) HMDS, (c) m-MTDATA (20 nm) films, and (d) NPD (30 nm).

\begin{tabular}{cccc}
\hline \hline Surface & $\begin{array}{c}\text { Mobility } \\
\left(\mathrm{cm}^{2} \mathrm{~V}^{-1} \mathrm{~s}^{-1}\right)\end{array}$ & $\begin{array}{c}\text { Threshold } \\
(\mathrm{V})\end{array}$ & $\begin{array}{c}\text { On/off } \\
\text { ratio }\end{array}$ \\
\hline $\mathrm{SiO}_{2}$ & 0.0075 & 66 & $6.6 \times 10^{4}$ \\
OTS & 0.50 & 23 & $5.8 \times 10^{6}$ \\
HMDS & 0.80 & 30 & $1.0 \times 10^{7}$ \\
$m$-MTDATA & 1.8 & 23 & $7.8 \times 10^{4}$ \\
NPD & 1.8 & 22 & $2.6 \times 10^{5}$ \\
\hline \hline
\end{tabular}

Table 2. The performances of the OFETs with various interfacial layers.

The mobility improvement can be caused also by change of the film structure. In this section, the film structures were evaluated by using atomic force microscopy (AFM) because 
$\mathrm{C}_{60}$ films deposited at room temperature generally shows no diffraction peak in XRD measurements. Figure 8 shows the morphology of the $\mathrm{C}_{60}$ films deposited on m-MTDATA and NPD interfacial layer, and those on the non-treated, OTS-treated and HMDS-treated substrates. The deposited film of $\mathrm{C}_{60}$ has granular surface with a diameter around $100 \mathrm{~nm}$, and almost no difference was observed for all the films. The root-mean-square (RMS) roughness of the $\mathrm{C}_{60}$ films on the m-MTDATA $(1.9 \mathrm{~nm})$ and NPD $(1.9 \mathrm{~nm})$ are almost the same with those on the non-treated substrate $(1.5 \mathrm{~nm})$, OTS-treated substrate $(1.5 \mathrm{~nm})$, and HMDS-treated substrate $(2.9 \mathrm{~nm})$. These results indicate that the observed improvement of electron mobility was not due to the morphological change of the $\mathrm{C}_{60}$ films.

(a)

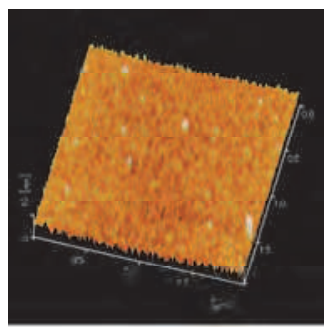

(d)

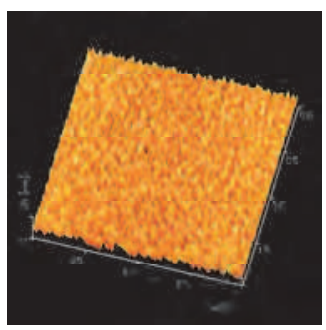

(b)

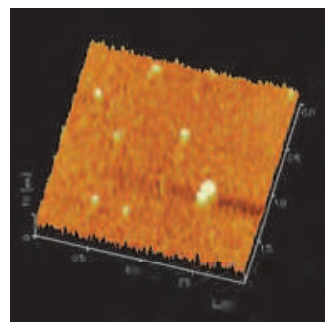

(c)

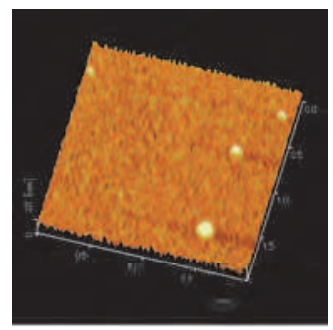

(e)

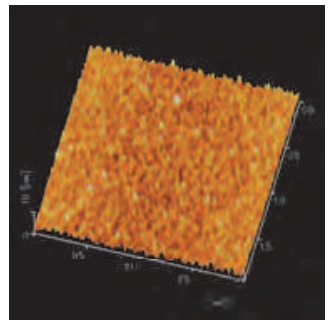

Fig. 8. The AFM images $\left(2 \times 2 \mu \mathrm{m}^{2}\right)$ of $\mathrm{C}_{60}$ deposited film $(100 \mathrm{~nm})$ on the various kinds of surface, (a) non-treated $\mathrm{SiO}_{2}$, (b) OTS, (c) HMDS, (d) m-MTDATA (20 nm), and (e) NPD $(30 \mathrm{~nm})$.

In the same manner as the previous section, the improvement by the hetero-layered structure of $\mathrm{C}_{60}$ OFET is attributed to the electronic effect at the interface. There is a large electron injection barrier from $\mathrm{C}_{60}(4.20 \mathrm{eV})$ to m-MTDATA $(1.90 \mathrm{eV})$ or NPD $(2.40 \mathrm{eV})$. Therefore, when the gate is positively biased, injected electrons from the source electrode would accumulate at the interface between $\mathrm{C}_{60}$ and hole transporting layer. Also in this case, the primary effect of the interfacial layer would be isolation of channel electrons from the $\mathrm{SiO}_{2}$ substrate surface having electron traps. This interpretation is supported by the fact that the threshold voltage becomes smaller (negatively shift) than that of non-treated device, which is similar to the effect of OTS and HMDS treatment. However, the hetero-layered OFETs with m-MTDATA showed higher mobility than that with OTS and HMDS treatment. This also means, electron donating character of hole transport layer and electron accepting character of $\mathrm{C}_{60}$ would cause partial electron transfer to fill the surface or interfacial traps in the $\mathrm{C}_{60}$ film. 
These effects also affect the air stability of n-type operation. It is well-known that n-type OFET is very sensitive to oxygen and water and does not work in air. Figure 9 shows the degradation characteristics of the field-effect mobility of $\mathrm{C}_{60}$ OFETs with exposure time to air. The device was placed in a dark box without any sealing under humidity of $30 \sim 40 \%$. The normal device with a bare surface showed rapid decrease of the field-effect mobility after exposure to air, and almost no operation was observed within 100 hours. The OTS treatment improved the initial performance; however, the degradation in air could not be prevented. On the other hand, the device of the hetero-layered OFETs with hole transporting layer showed much better stability. The device composed of NPD and $\mathrm{C}_{60}$ showed the mobility larger than $10^{-2} \mathrm{~cm}^{2} /$ Vs after 1000 hours exposure to air.

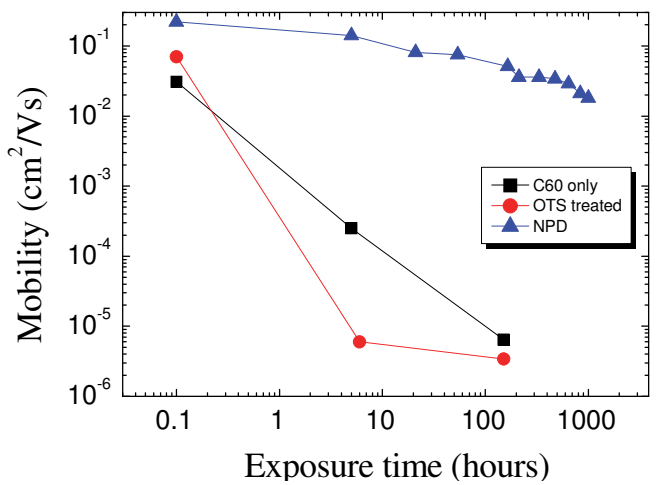

Fig. 9. Degradation characteristics of the field-effect mobility of $\mathrm{C}_{60}$ OFETs in air.

Generally, degradation of field-effect mobility in n-type OFET is interpreted as an increase of electron traps caused by oxygen or water at the channel layer or its interface with the insulator. In the same way as the initial performance, hole transporting layer having higher HOMO level gives electrons to $C_{60}$ in the ground state to fill the electron traps. Because the LUMO level of $\mathrm{C}_{60}$ is higher than the HOMO level of NPD, this charge transfer is partial and requires thermal activation. Therefore, additional oxygen or water by exposure to air would be compensated by the interfacial layer, resulting in long lifetime under atmospheric condition.

Thus, the concept of the hetero-layered OFET was extended to high performance n-type OFET using $\mathrm{C}_{60}$. Also in this case, the electron mobility was improved by the interfacial layer of NPD. In addition, the stability in air was drastically improved. These results also can be explained by partial electron transfer from the hole transporting layer to n-type channel layer leading to trap filling.

\section{Pentacene and electron transporting materials}

In this section, we extend the hetero-layered concept to the opposite combination of materials, that is, p-type organic semiconductor and electron transporting interfacial layer. For p-type semiconductor, pentacene was used as the most standard material. For the interfacial layer, many kinds of electron transporting materials were employed as shown in Figure 10. Most of them are electron transporting materials for OLED device forming amorphous film. The n-type organic semiconductor like NTCDA and $\operatorname{HAT}(\mathrm{CN})_{6}$ were also investigated. The HOMO and LUMO level of each material is shown in Figure 11. The 
materials are arranged by the LUMO levels representing electron accepting characters to discuss the energetic effects later.

The devices were fabricated in the same way and the device performance was measured in the glove box purged with dry nitrogen gas. The thickness of was $1.0 \mathrm{~nm}$ for the interfacial layer and $50 \mathrm{~nm}$ for the pentacene film.

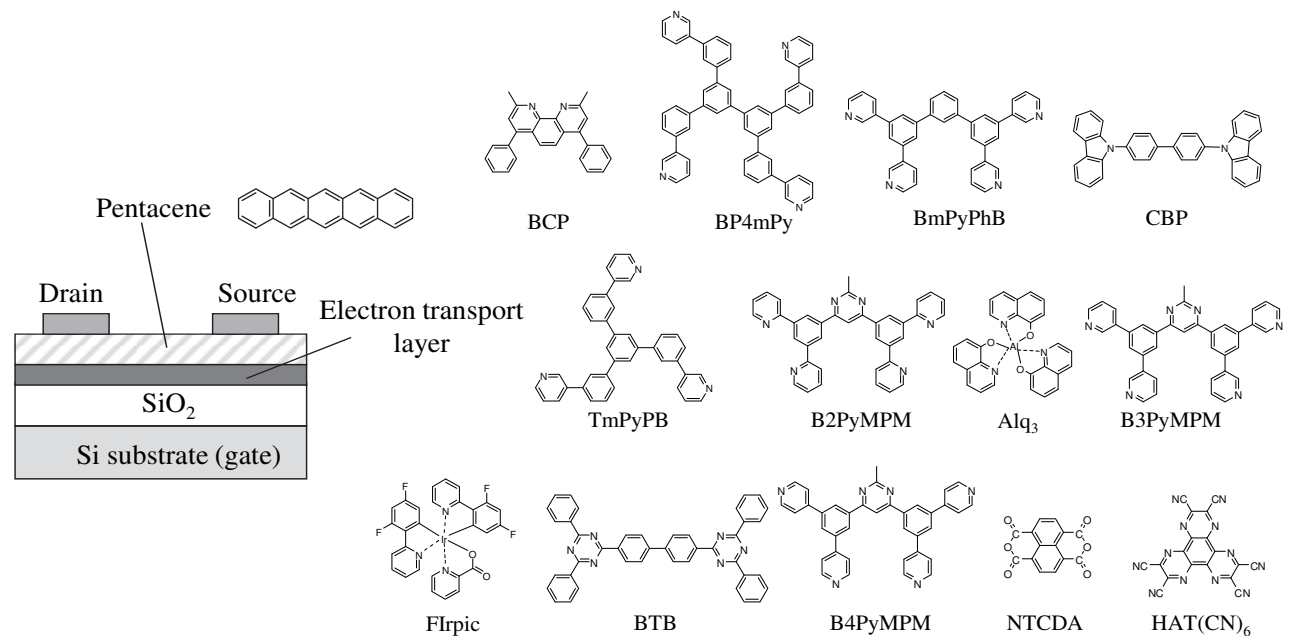

Fig. 10. Device structure of hetero-layered structure OFET using pentacene and electron transporting layer.

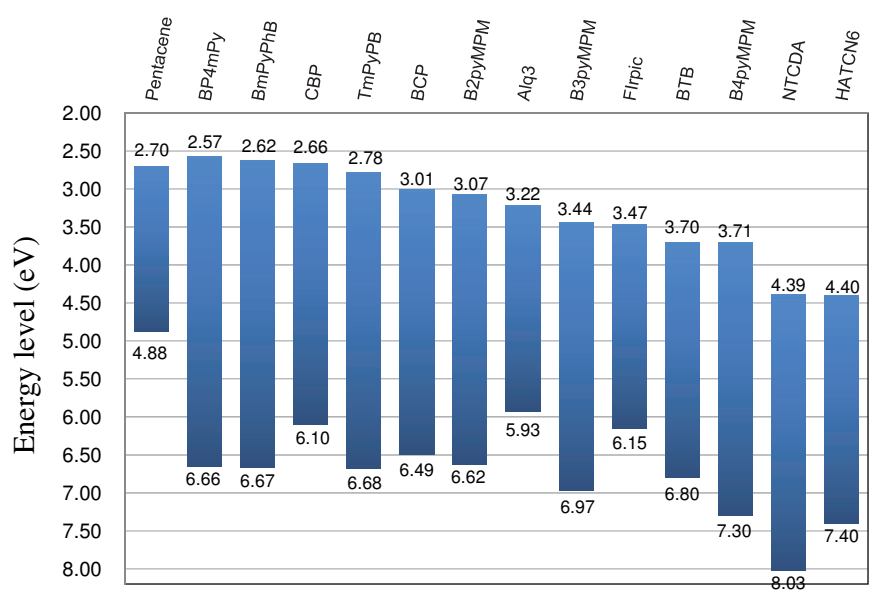

Fig. 11. Energy diagrams of the electron transporting materials used for interfacial layers.

The output curves of the hetero-layered OFETs using each interfacial layer were shown in Figure 12. All the devices showed pure p-type operation and no ambipolar operation was observed. The off current was decreased by inserting an interfacial layer of most electron transporting materials. On the other hand, in the case of NTCDA and HAT $(\mathrm{CN})_{6}$, the off current was increased and on/off ratio became lower. 


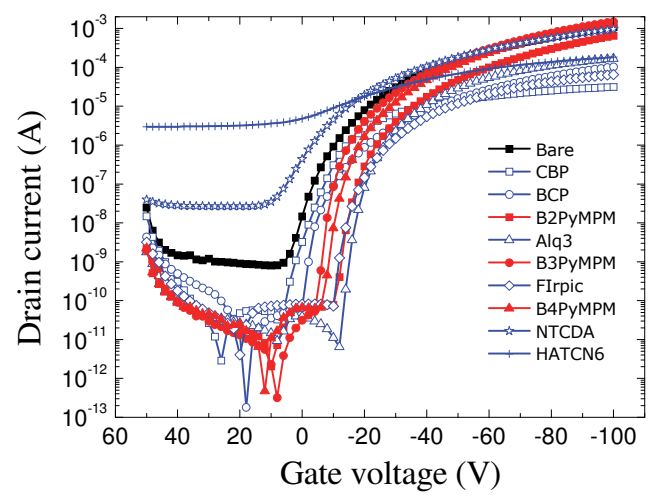

Fig. 12. Transfer curves of the heterolayered OFET composed of p-type pentacene and electron transporting interfacial layers.

\begin{tabular}{cccc}
\hline \hline Surface & $\begin{array}{c}\text { Mobility } \\
\left(\mathrm{cm}^{2} \mathrm{~V}^{-1} \mathrm{~s}^{-1}\right)\end{array}$ & $\begin{array}{c}\text { Threshold } \\
(\mathrm{V})\end{array}$ & $\begin{array}{c}\text { On/off } \\
\text { ratio }\end{array}$ \\
\hline Bare & 0.282 & -21 & $1.35 \times 10^{6}$ \\
BP4mPy & 0.276 & -28.7 & $1.89 \times 10^{9}$ \\
BmPyPhB & 0.361 & -31.8 & $6.13 \times 10^{10}$ \\
CBP & 0.019 & -4.7 & $1.09 \times 10^{7}$ \\
TmPyPB & 0.167 & -29.1 & $2.03 \times 10^{9}$ \\
BCP & 0.024 & -18.2 & $5.67 \times 10^{9}$ \\
B2PyMPM & 0.320 & -36.7 & $3.20 \times 10^{9}$ \\
Alq3 & 0.068 & -22.8 & $2.57 \times 10^{7}$ \\
B3PyMPM & 0.486 & -28.4 & $4.68 \times 10^{9}$ \\
Firpic & 0.019 & -18.6 & $1.61 \times 10^{7}$ \\
B4PyMPM & 0.388 & -29.1 & $2.57 \times 10^{9}$ \\
NTCDA & 0.193 & -11.3 & $3.61 \times 10^{4}$ \\
HAT(CN)6 & 0.033 & 10.7 & $6.05 \times 10^{1}$ \\
BTB & 0.045 & -14.8 & $1.48 \times 10^{9}$ \\
\hline \hline
\end{tabular}

Table 3. FET performance of the hetero-layered OFET composed of p-type pentacene and electron transporting interfacial layers.

The FET performance of each device is summarized in Table 3. The field-effect mobility was improved in some cases, and B3PyMPM showed the highest mobility of $0.486 \mathrm{~cm}^{2} / \mathrm{Vs}$ among these interfacial materials. The thickness of interfacial layers was $1 \mathrm{~nm}$ that is much thinner compared to the HTL/n-type layered OFET. In p-type hetero-layered device, thick interfacial layer $\sim 10 \mathrm{~nm}$ rather decreased the mobility in many cases. These results imply that role of the interfacial layer is different with n-type hetero-layered devices.

We discussed the correlation between the OFET performance and the LUMO level of the interfacial materials. Figure 13 shows the correlation between LUMO levels and field-effect mobility, and threshold voltages. From Fig. 13 (a), no correlation with the field-effect 
mobility was observed. These results imply the mobility is not determined by the electronic property of the interfacial material and charge transfer effect is not concerned. It would be because the hole transport is more stable and less affected by the interfacial traps compared to electron transport. On the other hand, weak correlation with threshold voltage was observed as shown in Fig. 13 (b). It was found that the threshold voltage becomes higher (positive shift) as the LUMO levels of the interfacial layer becomes lower (deeper). These results indicates that the charge transfer from pentacene to the electron transporting material promotes hole accumulation in the pentacene film to the gate voltage application. In the case of NTCDA and $\operatorname{HAT}(\mathrm{CN})_{6}$, their electron accepting character is so strong that hole doping occurred and off current was increased.

(a)

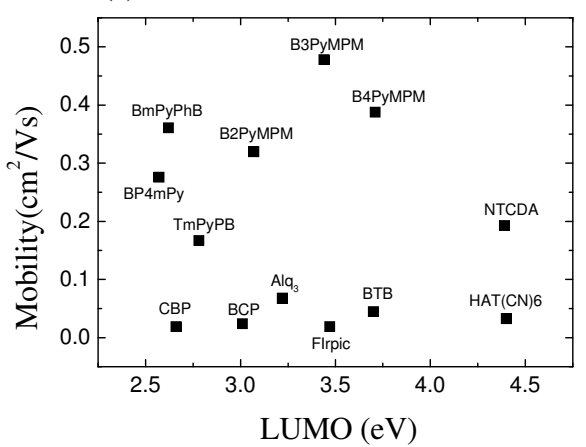

(b)

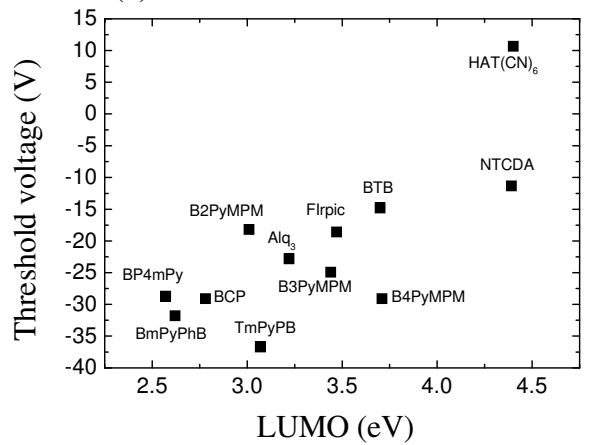

Fig. 13. Correlation between LUMO levels of interfacial electron transporting materials and (a) field-effect mobility, (b) threshold voltages.

The observed mobility can be explained by the film structure rather than energetic properties. Figure 14 shows the atomic force microscope (AFM) surface images of the thin pentacene film deposited on the interfacial layer. In the device showing high mobility, large and rigid granular domains were observed, for example, in the case of B3РуМРM, В4РуМРМ, $\mathrm{B} 2 \mathrm{PyMPM}$, and $\mathrm{BmPyPhB}$. On the other hand, small grains or amorphous-like surface were observed, for example, in the case of $\mathrm{Alq}_{3}$, FIrpic, and so on. From these results, we concluded that the mobility in the p-type hetero-layered OFETs composed of pentacene and electron transporting material is determined by the structural effects rather than the energetic effects.

The material group of B3PyMPM, B4PyMPM, B2PyMPM, and BmPyPhB showed large grains and higher mobility. These molecules were developed for electron transporting materials of OLED devices and very high performance was achieved (Tanaka et al., 2007; Sasabe et al., 2008). However, their LUMO levels are distributed from $3.71 \mathrm{eV}$ (B4PyMPM) to $2.62 \mathrm{eV}$ $(\mathrm{BmPyPhB})$. Therefore it is difficult to group these four materials by energetic properties, and electron accepting character leading to charge transfer seems to be not concerned. One plausible explanation is the molecular arrangement of the interfacial layer. These molecules include nitrogen atoms in the benzene ring. Since nitrogen atom has higher electron affinity that carbon atom, the nitrogen part become negatively charged. On the other hand, $\mathrm{SiO}_{2}$ surface without inert surface treatment has $\mathrm{OH}$ (hydroxyl) groups and its proton becomes positively charged. Therefore the nitrogen atoms in these molecules are attracted to the weak positive charge and the molecule would lie flat on the surface. This effect can be interpreted as electrostatic interaction between two point charges (nitrogen $\delta^{-}$and hydrogen $\delta^{+}$), rather than interaction between dipole moments between the molecule and hydroxyl group. In order to 
maximize Coulomb stabilization, all the nitrogen atoms should touch the surface, resulting in flat arrangement of the molecule on the surface. Consequently, very smooth and flat surface is achieved and pentacene film is expected to form high crystalline film with large grains.

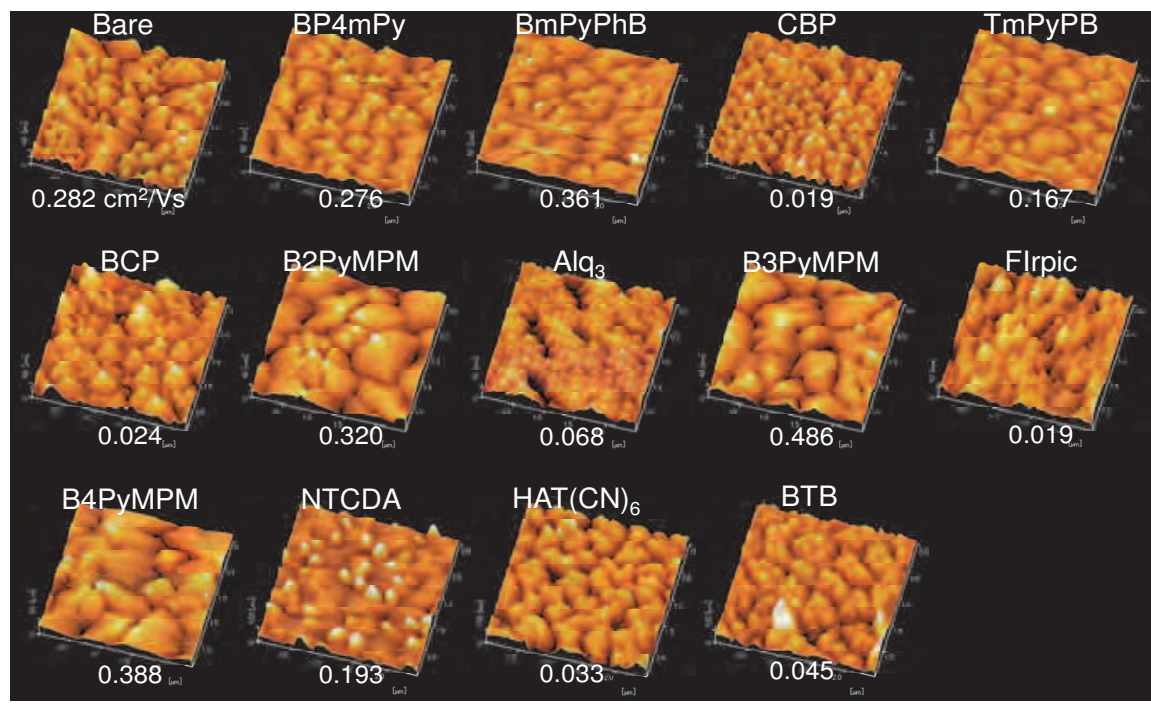

Fig. 14. AFM surface images of the pentacene film deposited on various interfacial layers.

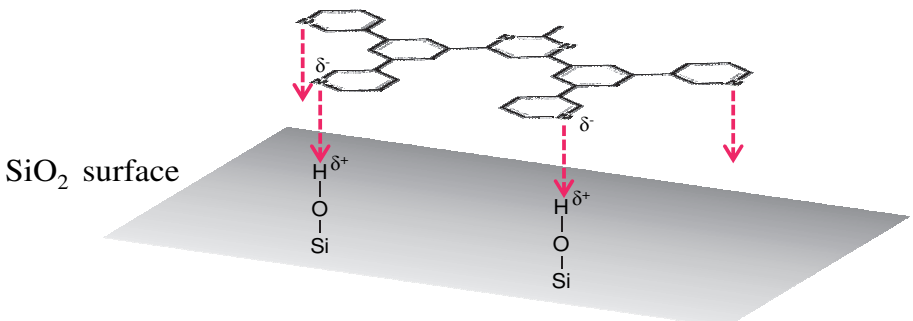

Fig. 15. Molecular arrangement model of B3PyMPM on the $\mathrm{SiO}_{2}$ surface.

Thus, the concept of heterolayered OFET was extended to p-type pentacene OFET with electron transporting interfacial layer. The performance was slightly improved, but it was mainly attributed to the effect of film structures. It was suggested that the electron transport material including nitrogen atoms forms a preferable underlayer to improve the crystallinity of the pentacene film on it.

\section{Conclusion}

In this chapter, we introduced a concept of heterolayered OFET composed of the channel organic semiconductor layer and the interfacial organic semiconductor having opposite polarity. In the HTL/n-type devices, the initial performance and stability in air was significantly improved. This effect can be attributed to electron transfer from HTL to n-type semiconductor at the interface, resulting in filling interfacial traps in advance. In the ETL/p- 
type devices, the performance was slightly improved, but that was mainly attributed to structural effect of film formation.

The hetero-layered OFET is very simple method. The device can be fabricated only by subsequent evaporation of two materials. It does not need self-assembly monolayer treatment taking a long time. Furthermore, it can be expected to solve the most serious problems in n-type OFET of mobility and stability in air. Our results suggest that air stable OFET without designing a new material having deep LUMO level. We expect a novel science and engineering for this "in-plane" carrier transport at the interface subjected to electrostatic gradient.

\section{Acknowledgement}

This study was partially supported by the New Energy and Industrial Technology Development Organization (NEDO), Precursory Research for Embryonic Science and Technology (PRESTO) program of the Japan Science and Technology agency (JST), and Grant-in-Aid for Scientific Research in Japan.

\section{References}

Chua, L. L., Zaumseil, J., Chang, J. F., Ou, E. C. W., Ho, P. K. H., Sirringhaus, H., and Friend, R. H., (2005) General observation of n-type field-effect behaviour in organic semiconductors, Nature Vol.434, No.7030, pp. 194-199, 193.

Dimitrakopoulos, C. D. and Malenfant, P. R. L., (2002) Organic thin film transistors for large area electronics, Adv. Mater. Vol.14, No.2, pp. 99-+, 510.

Lim, S. C., Kim, S. H., Lee, J. H., Kim, M. K., Kim, D. J., and Zyung, T., (2005) Surface-treatment effects on organic thin-film transistors, Synth. Met. Vol.148, No.1, pp. 75-79, 22.

Lin, Y. Y., Gundlach, D. J., Nelson, S. F., and Jackson, T. N., (1997) Stacked pentacene layer organic thin-film transistors with improved characteristics, IEEE Electron Device Lett. Vol.18, No.12, pp. 606-608, 189.

McCulloch, I., Heeney, M., Bailey, C., Genevicius, K., Macdonald, I., Shkunov, M., Sparrowe, D., Tierney, S., Wagner, R., Zhang, W., Chabinyc, M. L., Kline, R. J., McGehee, M. D., and Toney, M. F., (2006) Liquid-crystalline semiconducting polymers with high charge-carrier mobility, Nat Mater Vol.5, No.4, pp. 328-333, 1476-1122.

Rost, C., (2004) Ambipolar organic field-effect transistor based on an organic heterostructure, Vol.95, No.10, pp. 5782, 00218979.

Rost, C., Karg, S., Riess, W., Loi, M. A., Murgia, M., and Muccini, M., (2004) Ambipolar light-emitting organic field-effect transistor, Vol.85, No.9, pp. 1613, 00036951.

Sasabe, H., Chiba, T., Su, S. J., Pu, Y. J., Nakayama, K., and Kido, J., (2008) 2Phenylpyrimidine skeleton-based electron-transport materials for extremely efficient green organic light-emitting devices, Chem Commun (Camb) No.44, pp. 5821-5823, 1359-7345.

Singh, T. B., Sariciftci, N. S., Yang, H., Yang, L., Plochberger, B., and Sitter, H., (2007) Correlation of crystalline and structural properties of $\mathrm{C}[\mathrm{sub} 60]$ thin films grown at various temperature with charge carrier mobility, Vol.90, No.21, pp. 213512, 00036951.

Tanaka, D., Sasabe, H., Li, Y.-J., Su, S.-J., Takeda, T., and Kido, J., (2007) Ultra High Efficiency Green Organic Light-Emitting Devices, Vol.46, No.1, pp. L10-L12, 0021-4922 1347-4065.

Tatemichi, S., Ichikawa, M., Koyama, T., and Taniguchi, Y., (2006) High mobility n-type thin-film transistors based on N,N '-ditridecyl perylene diimide with thermal treatments, Appl. Phys. Lett. Vol.89, No.11, pp. 21. 


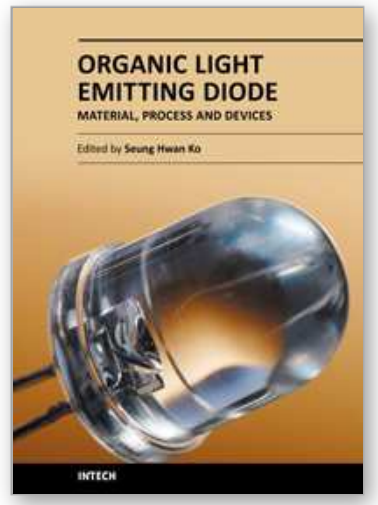

\author{
Organic Light Emitting Diode - Material, Process and Devices \\ Edited by Prof. Seung Hwan Ko
}

ISBN 978-953-307-273-9

Hard cover, 322 pages

Publisher InTech

Published online 27, July, 2011

Published in print edition July, 2011

This book contains a collection of latest research developments on Organic light emitting diodes (OLED). It is a promising new research area that has received a lot of attention in recent years. Here you will find interesting reports on cutting-edge science and technology related to materials, fabrication processes, and real device applications of OLEDs. I hope that the book will lead to systematization of OLED study, creation of new research field and further promotion of OLED technology for the bright future of our society.

\title{
How to reference
}

In order to correctly reference this scholarly work, feel free to copy and paste the following:

Ken-Ichi Nakayama, Masaaki Yokoyama, Yong-Jin Pu and Junji Kido (2011). Organic Field-Effect Transistors Using Hetero-Layered Structure with OLED Materials, Organic Light Emitting Diode - Material, Process and Devices, Prof. Seung Hwan Ko (Ed.), ISBN: 978-953-307-273-9, InTech, Available from:

http://www.intechopen.com/books/organic-light-emitting-diode-material-process-and-devices/organic-fieldeffect-transistors-using-hetero-layered-structure-with-oled-materials

\section{INTECH}

open science | open minds

\section{InTech Europe}

University Campus STeP Ri

Slavka Krautzeka 83/A

51000 Rijeka, Croatia

Phone: +385 (51) 770447

Fax: +385 (51) 686166

www.intechopen.com

\section{InTech China}

Unit 405, Office Block, Hotel Equatorial Shanghai

No.65, Yan An Road (West), Shanghai, 200040, China

中国上海市延安西路65号上海国际贵都大饭店办公楼 405 单元

Phone: +86-21-62489820

Fax: +86-21-62489821 
(C) 2011 The Author(s). Licensee IntechOpen. This chapter is distributed under the terms of the Creative Commons Attribution-NonCommercialShareAlike-3.0 License, which permits use, distribution and reproduction for non-commercial purposes, provided the original is properly cited and derivative works building on this content are distributed under the same license. 